\title{
sciendo
}

\section{Different Training Methods Cause Similar Muscle Damage in Youth Judo Athletes}

\author{
by \\ Antonijo Đerek ${ }^{1}$, Hrvoje Karninčić ${ }^{1}$, Emerson Franchini ${ }^{2}$, Saša Krstulović1, \\ Goran Kuvačić ${ }^{1}$
}

It is well known that different factors can contribute to muscle damage in judo matches or training. Previous research analyzed only the effects of simulated judo combat or judo training on biochemical markers of muscle damage without determining its specific causes. Our objective was to identify possible differences in biochemical markers of muscular damage in response to different training methods in youth judo athletes. Twelve high-level male judo athletes were randomly assigned to a standing ( $S P, n=6$, age $=16.6 \pm 1.1$ years) or a groundwork $(G P, n=6$, age $=17.8 \pm 0.8$ years) position combat practice group. Both groups had the same protocol of four 4-minute combat practice bouts separated by 1-minute rest intervals. Before and immediately after combat practice blood samples were taken to assess muscle damage markers: creatine kinase (CK), lactate dehydrogenase (LDH), aspartate aminotransferase (AST) and alanine aminotransferase (ALT). There were significant increases in AST, LDH, and CK after the standing and groundwork training sessions compared with resting values in both groups. Additionally, no significant differences in the enzyme's activity between SP and GP groups were found. These results showed that standing and groundwork randori training (free sparring or free practice) causes similar muscle damage in adolescent judo athletes. Future research should assess the effects of the same damage mechanisms over a longer period of time

Key words: biochemical markers, adolescent, exercise testing, judo, training.

\section{Introduction}

Intense physical activity causes damage to the muscle fibers of the striated muscle tissue, and high-intensity eccentric activities are considered to be one of the leading causes of muscle damage in the form of micro-damage at the cellular level (Hartmann and Mester, 2000). As eccentric activity results in muscle fiber elongation, the repetition of such activity consequently leads to damage at the junction of the two sarcomeres (Zline) and the leakage of intracellular metabolites into the intercellular spaces (Clarkson and Hubal, 2002). Additionally, muscle damage can also be induced by metabolic stress (Takarada, 2003) and mechanical impact (Wiechmann et al., 2016). Creatine kinase $(\mathrm{CK})$, lactate dehydrogenase
$(\mathrm{LDH})$, aspartate aminotransferase (AST) and alanine aminotransferase (ALT) enzymes are the most commonly used "leaky" products in the detection of muscle damage (Clarkson et al., 1992). These markers are not highly specific markers of damage to the striated muscle tissue because they can be found in other organs (e.g., heart muscle and liver) (Brancaccio et al., 2007).

Direct victory in judo is most often accomplished by one of the throwing techniques (Sterkowicz et al., 2013). However, the match can also end with the application of some of the techniques in the ground combat: pinning (osae komi waza), joint (kansetsu waza), and strangling (shime waza) techniques (Kano, 2013). Throwing techniques are considered to be complex judo-

1 - Faculty of Kinesiology, University of Split, Split, Croatia.

2 - Martial Arts and Combat Sports Research Group, Sport Department, University of Sao Paulo, Sao Paulo, Brazil. 
specific motor skills requiring powerful actions of short duration (1.0 - $1.4 \mathrm{~s})$ and include both lowerand upper-body muscle groups (Blais et al., 2007; Marcon et al., 2011). It is interesting to note that explosive eccentric-concentric contractions (Detanico et al., 2012) are applied to successful throwing techniques, which generates high mechanical loads, producing great stress in muscle structures (Detanico et al., 2015, 2017; Koga et al., 2013; Koshida et al., 2017). On the other hand, about $25 \%$ of valid judo match time is spent in groundwork (Castarlenas and Planas, 1997) characterized by a mix of whole-body dynamic and isometric actions involving muscle power, anaerobic-endurance and strengthendurance (Franchini et al., 2011, 2013).

It can be assumed that different stressors can contribute to muscle damage in a judo match, but very little is known about the causes of muscle damage. Specifically, in previous research, only the effects of simulated judo combat (Detanico et al., 2015; Ribeiro et al., 2006) or judo training (Detanico et al., 2017; Miura et al., 2005) on biochemical markers of muscle damage were analyzed, without determining its specific causes. Although no kicks are allowed in judo, when throwing techniques are executed, athletes collide with the ground with some impact force (Koshida et al., 2017). The magnitude of the impact, and thus the risk of muscle damage, will depend on the height of the fall and contact surface of the body with the ground (Bertocci et al., 2004).

During judo training, the main exercise used to improve both technical-tactical and physical conditioning of judo athletes is randori (free sparring or free practice) (Franchini and Takito, 2014), which is performed in its full version (i.e., standing and groundwork combats) or separated (i.e., standing or groundwork alone). The following randori frequency distribution was observed in Olympic medalists and nonmedalists: Medalists - complete: $30 \%$ performed 57 times per week; standing: $70 \%$ performed 5-7 times per week; groundwork: $50 \%$ performed 1-2 times per week; Non-medalists - complete: $27 \%$ performed 5-7 times per week; standing: $88 \%$ performed 5-7 times per week; groundwork: 45\% performed 3-4 times per week (Franchini and Takito, 2014). However, despite the fact that judo training involves possible different mechanisms of muscular damage and randori is performed basically in every judo session, little is known about the effects of isolated standing or groundwork randori on muscle damage. Therefore, the purpose of this paper was to identify possible differences in biochemical markers of muscular damage in response to standing and groundwork randori training.

\section{Methods}

\section{Participants}

Twelve $(n=12)$ high-level male judo athletes volunteered for this investigation. The athletes were national Championship medal holders in their age category. The data obtained after the experimental procedures showed abnormal values of CK serum activity in one subject. Additional investigation revealed that the subject had intense physical activity before this investigation. Therefore, he was excluded from further analysis. As the sample consisted of underage participants, written consent was obtained from parents/guardians after being thoroughly informed about potential risks and the purpose of this investigation. Each athlete had the full right to withdraw from the experiment at any time of testing. Because the present investigation required athletes to give their maximum effort, the following inclusion criteria were adopted: athletes had to be in exceptional health with no presence of cardiovascular disease, illness, injury, pain, and metabolic syndrome symptoms; to have a signed sport medical certification by a sports medicine specialist; uninterrupted training participation for at least six months (participation in at least $80 \%$ of the training sessions). The investigation was conducted in accordance with the 1975 Declaration of Helsinki ethical principles for scientific investigations and approved by the Ethical Committee of the Faculty of Kinesiology, University of Split (number: 2181-205-02-015-017; 16 July 2019).

\section{Experimental Design}

This was a comparative description (cross-sectional study), where the effects of two different training modalities (standing and groundwork randori) on the serum biomarkers of skeletal muscle damage (CK, LDH, AST, and ALT) were analyzed. The use of a single biomarker of skeletal muscle damage can result in an erroneous interpretation. Therefore, a multiple analysis was performed (Bessa et al., 2008). The 
randori was chosen as the training activity in order to allow all the judo actions. The groundwork combat is characterized by different movements on the mat, with the goal to successfully apply some of the osae komi waza, kansetsu waza, and shime waza. On the other hand, in a standing position, the goal is to throw the opponent with one of the throwing techniques (nage waza). From the point of view of possible muscular damage, the greatest difference in the two positions is that when sparring in a standing position, judo athletes throw each other and fall on the mat. However, if sparring is done by athletes with similar technical-tactical levels (as was the case in this investigation), it often happens that none of them succeeds in throwing the opponent. Therefore, to test the hypothesis, we had to modify the standing position combat practice by dividing it into 2 components: a) grip fighting, and b) explosive throwing techniques in default time frame. In this way, all subjects had an equal number of throws and falls with an intensity equal to a conventional randori.

The investigation was performed shortly after the competitive period was over. In the competitive period, athletes had an average training volume of $\sim 2 \mathrm{~h} /$ session, six days/week, mainly consisting of judo tactical training and some physical conditioning sessions. The training program was controlled and monitored by a professional judo coach.

Fifteen days before and upon their arrival, athletes were familiarized with testing procedures and briefly informed about the investigation aims. Athletes were asked not to perform any physical activity seven days prior to this investigation in order to prevent the most common causes of muscle damage, such as injuries, direct hits, and strenuous physical exercise (Brancaccio et al., 2010). Additionally, to reduce any interference in baseline results, athletes were requested to refrain from drinking caffeine-containing beverages, vitamins, or supplements for $24 \mathrm{~h}$ before and during the experiment. Athletes were not involved in any rapid weight loss procedures in the month of this experiment.

\section{Procedures}

Athletes were matched according to their body mass, with a difference of no more than $15 \%$ between them. Pairs of similar body mass were then randomly assigned to the standing (SP) or the groundwork position (GP) combat practice group. After group allocation, athletes performed a 20 min general warm-up composed of jogging, walking, and stretching with some judo-specific exercises without falling and throwing techniques.

The SP group performed four 4-minute combat practice bouts separated by one 1-minute rest intervals. Athletes were instructed to intermittently throw each other every $10 \mathrm{~s}$ (grip fighting between each throw), resulting in a total of 96 nage-komi (throwing technique exercise), with each athlete performing 48 throws. The GP group performed combat practice in the same temporal structure (four 4-minute combat practice bouts separated by 1-minute rest intervals). Athletes were instructed to continue the practice in the event of groundwork technique, which would normally occur at the end of a match (ippon).

All experimental procedures were conducted by sport and exercise scientists, members of the Faculty of Kinesiology, University of Split, together with the certified medical technician and physician from Medical Biochemistry Laboratory, LabPlus Split, Croatia. Standing and groundwork randori were performed at a local judo club (ambient conditions: $\sim 24^{\circ} \mathrm{C}, 30 \%$ relative humidity) on a judo mat as recommended by the International Judo Federation (IJF) with a density of $240 \mathrm{~kg} / \mathrm{m}^{3}$. Venous blood samples were taken before and after the randori (10 minutes after the last round) for serum AST, ALT, LDH, and CK activity analysis.

\section{Evaluation of serum biomarkers}

Blood samples $(4 \mathrm{~mL})$ were obtained from the cubital vein before (pre) and immediately after (post) combat practice and stored in a biochemical tube without anticoagulants. The extraction of a blood sample was performed while the athletes were seated. Samples were left at room temperature for $30 \mathrm{~min}$ to cool and coagulate. Blood samples were then centrifuged at $3500 \mathrm{rpm}$ for 10 minutes to extract blood serum from which the activities of biochemical markers were subsequently determined. All samples were analyzed using a Beckman Coulter Olympus AU480 biochemical analyzer based on the recommendations of the International Federation of Clinical Chemistry (IFCC). The average intra- 
assay reliability expressed as coefficients of variation, was $1 \%$ for $\mathrm{CK}, 0.89 \%$ for $\mathrm{LDH}, 1.93 \%$ for AST, and $2.95 \%$ for ALT.

Heart rate and session rating of perceived exertion measurements

Objective (heart rate) and subjective (session rating of perceived exertion) variables of effort were assessed to quantify intensity achieved during the experiment. With the short-range radio telemetry monitor (Polar S810, OY Finland), the heart rate (HR) was continuously recorded at $5 \mathrm{~s}$ intervals. The HR monitors were fixed on the chest of athletes by an adjustable elastic strap. In this way, HR recording errors were minimized. Additionally, athletes were asked to re-check HR monitors before each combat practice bout. Thirty minutes after the last practice bout, athletes were asked to rate their perceived exertion (sRPE) during their experiment. Athletes were asked to evaluate their workout using the Borg Category Ratio-10 (CR-10) RPE scale modified by Foster et al. (2001).

\section{Statistical Analysis}

The normality of data was confirmed using the Kolmogorov-Smirnov test, therefore the results were expressed as the mean and standard deviation. However, considering the small sample size of both groups, to determine the differences in biomarkers of skeletal muscle damage between the SP and GP groups in each time-point (pre, post), the Mann-Whitney U-Test was used. Furthermore, to determine the differences in each time point (pre, post) for each group, the Wilcoxon signed-ranks test was used. Effect sizes were calculated as $r=Z /(\sqrt{ } \mathrm{Nobs})$, with $0.10,0.30$, and 0.50 regarded as a small, medium, and large effects, respectively (Cohen, 1992). The level of significance $(p)$ was set at 0.05 . All statistical analyses were performed using commercial software Statistica ver. 13.5 (Dell Inc., Round Rock, TX USA).

\section{Results}

Table 1 shows the anthropometric and sRPE measurements.

The average HR during combat practices showed no significant differences between standing and ground positions $(z=1.1, p=0.27$, $E S=-0.33$, medium). Additionally, we found no significant differences in sRPE between groups $(z$ $=-0.31, p=0.76, E S=-0.07$, small).
Figure 1 (Panel A) shows changes in AST serum acitvity before and after combat practice. Activity significantly increased after combat practice in the SP $(z=2.06, p=0.04, E S=-0.65$, large $)$ and the GP $(z=2.21, p=0.03, E S=-0.64$, large) group. The Mann-Whitney U Test showed significant difference between the groups in pre $(z=2.21, p=$ $0.03, E S=-0.67$, large $)$ and post $(z=2.22, p=0.03$, $E S=-0.67$, large) randori. There were no significant differences in ALT activity between groups, nor were any significant differences in pre and post randori (Figure 1, Panel B). The baseline and post testing serum activity of $\mathrm{LDH}$ are presented in Figure 1, Panel C. When compared to baseline values, serum LDH increased significantly after the combat practice in the SP $(z=2.03, p=0.04$, ES $=-0.64$, large $)$ and the GP $(z=2.2, p=0.03, E S=-$ 0.64 , large) group. Similar results can be observed for CK activity, where post randori data were significantly different when compared to baseline values in both SP $(z=2.02, p=0.04, E S=-0.64$, large) and GP $(z=2.2, p=0.03, E S=-0.64$, large $)$ groups (Figure 1, Panel D). We found no significant differences in LDH and CK serum activity between groups in each time point.

\section{Discussion}

The primary question of this investigation was whether there were any effects of different modalities of judo training on biochemical markers of muscular damage. Therefore, two training modalities were used to investigate muscular damage variables: standing and groundwork combat practices. To the best of the authors' knowledge, no one performed an investigation with this approach in adolescent judo athletes, especially using a long-interval (i.e., 168 hours) between the last training and the experimental conditions. The main finding of our study was that there were significant increases in AST, LDH, and CK activity for both groups after the training session compared with resting values. Additionally, although subjects in the SP group were involved in training designed to include more possible causes of biochemical markers in the intercellular space (more pronounced eccentric leg movements, throws, and falls, accelerated metabolic processes) compared to the GP group (dominant isometric conditions), no statistically significant differences in the activity of the enzymes between the groups were found, 
except for AST, which was higher for the GP group at rest and after the training session compared with the SP group.

The basal values of serum activity suggest that one week of rest was sufficient to return the enzymatic activity values to normal (ref. intervals $\left(\mathrm{U} \cdot \mathrm{L}^{-1}\right)$ for CK are 70 - 285; for AST 11 - 38, and for ALT 10 - 33) with LDH activity at the upper limit of the reference values (127-231 U.L-1). As athletes may have elevated basal values of the measured enzymes relative to non-athletes (Brancaccio et al., 2010), the results likely suggest adaptation of the organism to frequent work with high glycolytic activation because LDH catalyzes the conversion of pyruvate to lactate and back (Brancaccio et al., 2010).

As this is the first study that used isolated standing and groundwork combat simulations to verify their effects on muscle damage markers, the comparison with the judo-specific literature is difficult. In fact, only few studies analyzed the muscle damage markers during judo-specific combat (Detanico et al., 2015; Franchini et al., 2016) or traditional judo training sessions (Detanico et al., 2017; Koga et al., 2013). Detanico et al. (2015) reported increased CK and LDH activity after three 5-min judo matches interspersed with 15-min passive recovery intervals compared to pre-match values, whereas Franchini et al. (2016) observed increased ALT, AST, LDH, and CK activity after a single 5-min judo match compared to pre-match values. These results are similar to those found in the present study, except for the increased ALT observed in the study by Franchini et al. (2016). When traditional judo training sessions were analyzed, Koga et al. (2013) observed increased CK activity after a 2-h judo training session composed of a 15min warm-up, a 20-min uchi-komi (technique repetition), a 70-min randori, and a 15-min cooldown. Detanico et al. (2017) used a shorter training session (90 min) composed of a 15-min warm-up, 35-min technical training (including uchi-komi and nage-komi, throwing technique repetitions) and a 40-min randori (including four 2-min groundwork combats interspersed with 2min rest intervals and four 3-min standing combats interspersed with 3-min rest intervals) and also revealed increased CK activity after the training sessions compared to pre-training sessions values. Therefore, isolated or combined standing and groundwork judo combats, as well as traditional judo training sessions, result both in muscle damage.

\begin{tabular}{|c|c|c|c|c|c|c|}
\hline \multicolumn{7}{|c|}{ Table 1} \\
\hline Group & Age (years) & $\begin{array}{c}\text { Experience } \\
\text { (years) }\end{array}$ & $\begin{array}{l}\text { Body height } \\
\text { (cm) }\end{array}$ & $\begin{array}{c}\text { Body mass } \\
(\mathrm{kg})\end{array}$ & HR (bpm) & sRPE (a.u.) \\
\hline $\mathrm{SP}(\mathrm{n}=5)$ & $16.6 \pm 1.1$ & $8.8 \pm 1.8$ & $178.8 \pm 5.1$ & $76.2 \pm 1.8$ & $194.6 \pm 6.8$ & $7.5 \pm 0.3$ \\
\hline $\mathrm{GP}(\mathrm{n}=6)$ & $17.8 \pm 0.8$ & $8.7 \pm 2.9$ & $180.3 \pm 8.0$ & $80.7 \pm 6.6$ & $182.7 \pm 19.2$ & $7.6 \pm 0.8$ \\
\hline \multicolumn{7}{|c|}{$\begin{array}{l}S P \text { - standing position group; GP - groundwork position group; } \\
H R \text { - heart rate; sRPE - session rating of perceived exertion }\end{array}$} \\
\hline
\end{tabular}



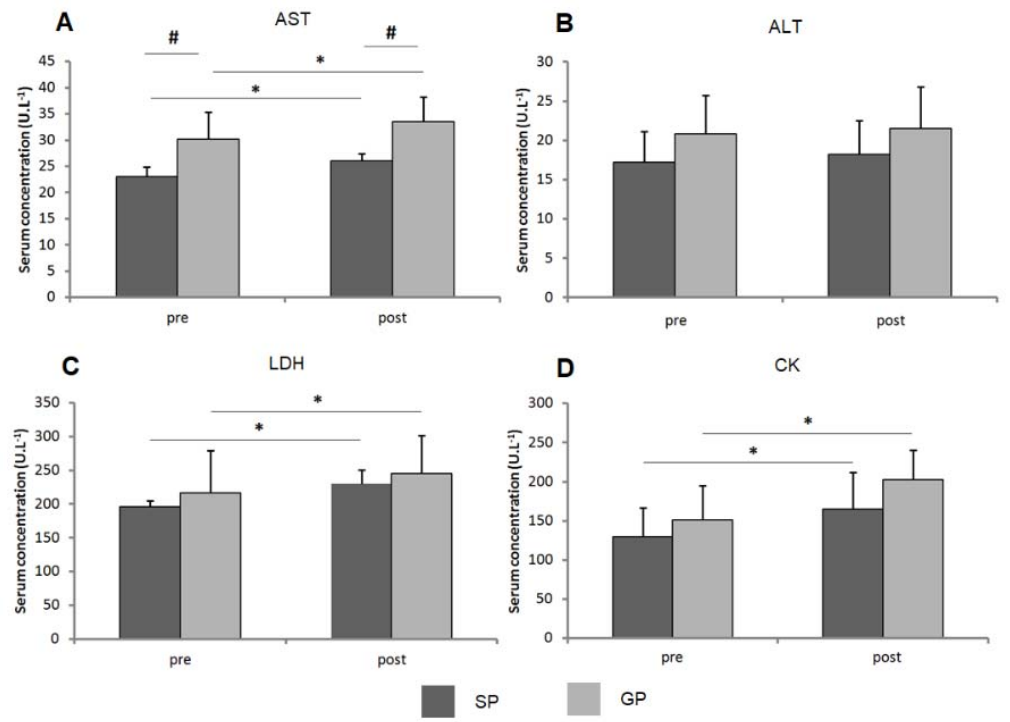

\section{Figure 1}

Aspartate aminotransferase (AST, Panel A), alanine aminotransferase (ALT, Panel B), lactate dehydrogenase ( $L D H$, Panel $C)$, and creatine kinase (CK, Panel $D)$ activity pre and post combat practice during standing (SP) and groundwork (GP) position. ${ }^{*}$ denotes significant differences between pre and post combat practice $(p<0.05)$; \# denotes significant differences between groups in pre and post combat practice $(p<0.05)$.

A possibility to compare the responses under the groundwork condition of our study with other studies is to consider the investigations focused on Brazilian jiu-jitsu, a grappling combat sport that utilizes techniques similar to judo, but which is performed basically under the groundwork condition (Andreato et al., 2012, 2015; Branco et al., 2016; Fonseca et al., 2016; Pinho Júnior et al., 2014; Santos et al., 2012). One study with Brazilian jiu-jitsu indicated that a single 7-min combat resulted in increases in ALT and LDH, but not in CK and AST (Andreato et al., 2012). Conversely, Andreato et al. (2016) compared match simulations lasting 2-min, 5-min, 8-min, and 10-min and reported no changes in CK, AST, and ALT, but increased LDH activity after 8-min matches compared to resting values. Additionally, Andreato et al. (2012) investigated muscle damage caused by simulated Brazilian jiu- jitsu competition (four 10-min matches interspersed with 10-min rest intervals) and indicated that CK activity was higher after postmatch 4 compared to pre-match 1 , post-match 1 and pre-match 2, whereas AST was lower at rest than after each match, and ALT at rest was lower than post-match 3 , with no significant changes in $\mathrm{LDH}$. Therefore, for groundwork combats, there is a higher variation in the results reported in the literature, suggesting that more studies are needed to understand factors contributing to specific muscle damage marker changes. However, at least one muscle damage marker increased after the match or competition simulations, confirming the results of the present studies and suggesting that more than one muscle damage marker should be monitored to identify this phenomenon properly.

Two studies analyzed the effects of judo- 
related training on muscle damage responses to a traditional judo training session (Koga et al., 2013) and a 5-min judo match simulation (Franchini et al., 2016). After three months of judo-specific training, Koga et al. (2013) indicated that judo athletes presented a lower CK elevation after a 2-h judo training $(7.4 \pm 7.8 \%)$ session compared to pre-training $(30.9 \pm 15.7 \%)$. Additionally, before the training period, CK activity increased $24 \mathrm{~h}$ after training $(79.3 \pm 98.0 \%)$ compared to resting values, whereas post-training CK activity did not differ between the values reached $24 \mathrm{~h}$ after training compared to resting values $(-6.8 \pm 17.8 \%)$, indicating that judo athletes adapt to the judospecific stress and decreased muscle damage is observed after only a 3-month training period. Franchini et al. (2016) submitted judo athletes to different high-intensity interval training modes (upper-body, lower-body, and uchi-komi during 4 weeks, two sessions per week, added to the traditional judo training sessions) and reported that CK activity decreased after the match simulation in the post-training period compared to the pre-training period for the upper-body training group, but AST, LDH, and ALT were not affected by the short-term low-volume highintensity interval training intervention. Therefore, only one muscle damage marker decreased after the training intervention and this occurred only for the group submitted to the upper-body exercise mode, which is the main muscle group involved during judo grip dispute and technique unbalance actions.

\section{Conclusions}

In summary, there were significant increases in AST, LDH, and CK activity for both groups after the standing and groundwork training sessions compared with baseline values, with no difference between training modes. Therefore, considering that muscle damage is similar between these two conditions, judo coaches can choose a specific training mode (i.e., standing, groundwork, or both together) based on technical-tactical needs, whereas muscle damage recovery can be addressed considering a similar approach for both conditions. Indeed, the dynamics of measured enzymes in recovery needs attention, which is also the major drawback of this study. Different damage mechanisms lead to different recovery mechanisms, which are ultimately manifested in different retention times of the measured enzymes in the intercellular space (Brancaccio et al., 2010). Therefore, future research should compare different damage mechanisms over a longer time.

\section{Acknowledgements}

The authors wish to thank all young athletes for their enthusiastic participation in this investigation. No financial or material support of any kind was received for the work described in this article

\section{References}

Andreato LV, de Moraes SMF, del Conti Esteves JV, Pereira RRDA, Gomes TLDM, Andreato TV, Franchini E. Physiological responses and rate of perceived exertion in brazilian jiu-jitsu athletes. Kinesiology, 2012; 44(2): 173-181

Andreato LV, Julio UF, Panissa VLG, del Conti Esteves JV, Hardt F, de Moraes SMF, de Souza CO, Franchini E. Brazilian jiu-jitsu simulated competition part I: metabolic, hormonal, cellular damage, and heart rate responses. J Strength Cond Res, 2015; 29(9): 2538-2549

Andreato LV, del Conti Esteves JV, Julio UF, Gonçalves Panissa VL, Hardt F, Pastório EJ, de Moraes SMF, Franchini E. Metabolic, muscle damage and heart rate responses in Brazilian jiu-jitsu matches of varied duration. Kinesiology: International journal of fundamental and applied kinesiology, 2016; 48(2): 182192

Bertocci GE, Pierce MC, Deemer E, Aguel F, Janosky JE, Vogeley E. Influence of fall height and impact surface on biomechanics of feet-first free falls in children. Injury, 2004; 35(4): 417-424

Bessa A, Nissenbaum M, Monteiro A, Gandra PG, Nunes LS, Bassini-Cameron A, Werneck-de-Castro JP, de Macedo DV, Cameron LC. High-intensity ultraendurance promotes early release of muscle injury markers. Br J Sports Med, 2008; 42(11): 589-593

Blais L, Trilles F, Lacouture P. Three-dimensional joint dynamics and energy expenditure during the execution of a judo throwing technique (Morote Seoï Nage). J Sports Sci, 2007; 25(11): 1211-1220 
Brancaccio P, Lippi G, Maffulli N. Biochemical markers of muscular damage. Clin Chem Lab Med, 2010; 48(6): 757-767

Brancaccio P, Maffulli N, Limongelli FM. Creatine kinase monitoring in sport medicine. Br Med Bull, 2007; 81-82: 209-230

Branco BHM, Fukuda DH, Andreato LV, Santos JF, del Conti Esteves JV, Franchini E. The effects of hyperbaric oxygen therapy on post-training recovery in jiu-jitsu athletes. PLoS One, 2016; 11(3): e0150517

Castarlenas J, Planas A. Study of the temporal structure of judo combat. Educ Física y Deport, 1997; 1(47): 32 39

Clarkson PM, Hubal MJ. Exercise-induced muscle damage in humans. Am J Phys Med Rehabil, 2002; 81(11 Suppl): 52-69

Clarkson PM, Nosaka K, Braun B. Muscle function after exercise-induced muscle damage and rapid adaptation. Med Sci Sports Exerc, 1992; 24(5): 512-520

Cohen J. Statistical Power Analysis. Curr Dir Psychol Sci, 1992; 1(3): 98-101

Detanico D, Dal Pupo J, Franchini E, dos Santos SG. Effects of successive judo matches on fatigue and muscle damage markers. J Strength Cond Res, 2015; 29(4): 1010-1016

Detanico D, Dal Pupo J, Franchini E, dos Santos SG. Relationship of aerobic and neuromuscular indexes with specific actions in judo. Sci Sports, 2012; 27(1): 16-22

Detanico D, Dal Pupo J, Franchini E, Fukuda DH, dos Santos SG. Effects of traditional judo training session on muscle damage symptoms. J Sports Med Phys Fitness, 2017; 57(6): 872-878

Fonseca LB, Brito CJ, Silva RJS, Silva-Grigoletto ME, da Silva WM Junior, Franchini E. Use of cold-water immersion to reduce muscle damage and delayed-onset muscle soreness and preserve muscle power in jiu-jitsu athletes. J Athl Train, 2016; 51(7): 540-549

Foster C, Florhaug JA, Franklin J, Gottschall L, Hrovatin LA, Parker S, Doleshal P, Dodge C. A new approach to monitoring exercise training. J Strength Cond Res, 2001; 15(1): 109-115

Franchini E, Artioli GG, Brito CJ. Judo combat: Time-motion analysis and physiology. Int J Perform Anal Sport, 2013; 13(3):6 24-641

Franchini E, Del Vecchio FB, Matsushigue KA, Artioli GG. Physiological profiles of elite judo athletes. Sport Med, 2011; 41(2): 147-166.

Franchini E, Julio UF, Gonçalves Panissa VL, Lira FS, Agostinho MF, Branco BHM. Short-term low-volume high-intensity intermittent training improves judo-specific performance. Arch Budo, 2016; 12: 219-229

Franchini E, Takito MY. Olympic preparation in Brazilian judo athletes: Description and perceived relevance of training practices. J Strength Cond Res, 2014; 28(6): 1606-1612

Hartmann U, Mester J. Training and overtraining markers in selected sport events. Med Sci Sports Exerc, 2000; 32(1): 209-215

Kano J. Kodokan Judo: The Essential Guide to Judo by Its Founder Jigoro Kano. Reprint ed. Kodansha International; 2013

Koga T, Umeda T, Kojima A, Tanabe M, Yamamoto Y, Takahashi I, Iwasaki H, Iwane K, Matsuzaka M, Nakaji S. Influence of a 3-month training program on muscular damage and neutrophil function in male university freshman judoists. Luminescence, 2013; 28(2): 136-142

Koshida S, Ishii T, Matsuda T, Hashimoto T. Biomechanics of judo backward breakfall for different throwing techniques in novice judokas. Eur J Sport Sci, 2017; 17(4): 417-424

Marcon G, Franchini E, Jardim JR, Barros Neto TL. Structural analysis of action and time in sports: judo. J Quant Anal Sport, 2010; 6(4)

Miura M, Umeda T, Nakaji S, Liu Q, Tanabe M, Kojima A, Yamamoto Y, Sugawara K. Effect of 6 months' training on the reactive oxygen species production capacity of neutrophils and serum opsonic activity in judoists. Luminescence 2005; 20(1): 1-7

Pinho Júnior EA, Brito CJ, Santos WOC, Valido CN, Mendes EL, Franchini E. Influence of cryotherapy on muscle damage markers in jiu-jitsu fighters after competition: A cross-over study. Rev Andaluza Med del Deport, 2014; 7(1): 7-12 
Ribeiro SR, Tierra-Criollo CJ, Martins RÁBL. Effects of different strengths in the judo fights, muscular electrical activity and biomechanical parameters in elite athletes. Rev Bras Med do Esporte, 2006; 12(1): 27-32

Santos WOC, Brito CJ, Pinho Júnior EA, Valido CN, Mendes EL, Nunes MAP, Franchini E. Cryotherapy post-training reduces muscle damage markers in jiu-jitsu fighters. J Hum Sport Exerc, 2012; 7(3): 629638

Sterkowicz S, Sacripanti A, Sterkowicz-Przybycien K. Techniques frequently used during London Olympic judo tournaments: a biomechanical approach. Arch Budo, 2013; 9(1): 51-58

Takarada Y. Evaluation of muscle damage after a rugby match with special reference to tackle plays. $\mathrm{Br} J$ Sports Med, 2003; 37(5): 416-419.

Wiechmann GJ, Saygili E, Zilkens C, Krauspe R, Behringer M. Evaluation of muscle damage marker after mixed martial arts matches. Orthop Rev, 2016; 8(1): 1-4

\section{Corresponding author:}

\section{Goran Kuvačić, PhD}

Faculty of Kinesiology, University of Split, Teslina 6, 21000 Split, Croatia

Phone: 00385951979970

E-mail address: gorkuv@kifst.hr 